\title{
Tecnécio-99M-MIBI no acompanhamento de carcinoma diferenciado de tireóide
}

\author{
Technetium-99m-MIBI in the follow-up of differentiated thyroid carcinoma
}

Luiz S. Machado', Dauro S.V. Pedras ${ }^{2}$, Jane B. Antonucci ${ }^{3}$, Rossana Corbo ${ }^{4}$

\section{Resumo}

Tecnécio-99m-metoxiisobutil isonitrila ( ${ }^{99 m} \mathrm{~T} \mathrm{c-M} \mathrm{IBI)} \mathrm{foi,} \mathrm{recentemente,} \mathrm{introduzido} \mathrm{como} \mathrm{fármaco} \mathrm{marcador-tu-}$ moral. Este estudo avalia o papel da cintilografia com ${ }^{99 m} \mathrm{~T}$ c-M IBI na localização de lesões metastáticas através da análise de sua sensibilidade e especificidade, em comparação com rastreamento com ${ }^{131}$ e níveis séricos de tireoglobulina. Foram estudados oitenta e nove pacientes tireoidectomizados por câncer diferenciado de tireóide. Foram adquiridas imagens de corpo inteiro precoces (20 minutos) e tardias (6 e 24 horas) após injeção de 99mT c$\mathrm{MIBI}$. Foram determinados os níveis séricos de tireoglobulina durante tratamento com hormônio tireoideano e 4 semanas após sua suspensão; rastreamento com ${ }^{131}$ | foi realizado após dose diagnóstica ou terapêutica/ablativa. O s pacientes que apresentaram resultados discordantes, foram submetidos a confirmação diagnóstica por tomografia computadorizada ou ressonância magnética. No grupo com rastreamento com ${ }^{131}$ | negativo (34/89), 23 pacientes apresentaram cintilografia com ${ }^{99 m}$ T c-M IBI negativa e 11 pacientes tiveram resultado discordante (especificidade: 67,6\%); 22 pacientes apresentaram cintilografia com ${ }^{99 m T ~ c-M ~ I B I ~ e ~ r a s t r e a m e n t o ~ c o m ~}{ }^{131}$ positivas; enquanto 0 total de rastreamento com ${ }^{131}$ positivos foi 55 (sensibilidade: $40,0 \%$ ). O nze pacientes apresentaram rastreamento com $^{131}$ I e cintilografia com ${ }^{99 m} \mathrm{~T} \mathrm{C-M} \mathrm{IBI}$ positiva; 7 destes pacientes foram comprovados como verdadeiros positivos; e quando associados os valores de tireoglobulina e os resultados encontrados pela cintilografia com ${ }^{99 \mathrm{mT}} \mathrm{C}-\mathrm{M} \mathrm{IBI}$, a

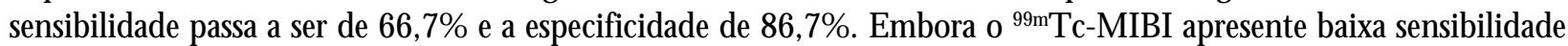

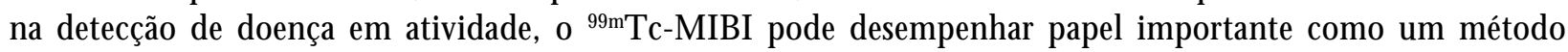
complementar aos valores de tireoglobulina e rastreamento com ${ }^{131}$.

Palavras-chave: N eoplasias da tireóide; Carcinoma; Cintilografia; Tireoglobulina; M IBI.

\footnotetext{
${ }^{1}$ M édico especializando do curso de M edicina N uclear- Seção de M edicina N uclear do H ospital do Câncer I-IN CA

2 M édico Seção de M edicina Nuclear do H ospital do Câncer I-IN CA

${ }^{3}$ Prof. Chefe Seção de M edicina N uclear do H ospital do Câncer I-INCA

${ }^{4}$ Profa. D ra . da disciplina de Endocrinologia da U niversidade Federal do Rio de Janeiro, endocrinologista da Seção de M edicina N uclear do H ospital do Câncer I-INCA

Endereço para correspondência: Imachado@mac.com; jane@inca.gov.br
} 


\begin{abstract}
Technetium-99m-methoxyisobutil isonitrila ( ${ }^{99 \mathrm{mT}} \mathrm{c}-\mathrm{M} \mathrm{IBI}$ ) has been introduced as a tumor-seeking agent in the past few years. The present study has evaluated the role of ${ }^{99 m T}$ c-M IBI whole body scan (W BS) in localizing metastatic lesions by assessing sensitivity and specificity in comparison with ${ }^{131}$ scan and serum thyroglobulin measurement. Eighty-nine thyroidectomized patients due to differentiated thyroid cancer were enrolled. Early (20 minutes) and

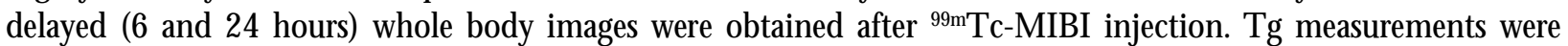
obtained during thyroid hormone reposition and 4 weeks after hormone discontinuation, ${ }^{131}{ }^{1}$ scans were performed after low diagnostic or high ablative/therapeutic dose. These methods were used as standard to evaluate the

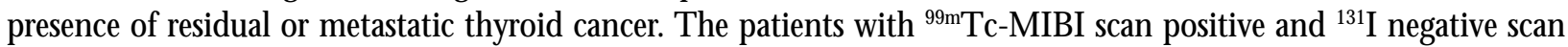
underwent chest x-ray, computed tomography (CT) or magnetic resonance imaging (M RI). In the group with ${ }^{131}$ negative scan (34/89), 23 patients presented ${ }^{99 m}$ T c-M IBI W BS negative and 11 patients were discordant (specificity: $67,6 \%$ ), and 22 patients had ${ }^{99 m}$ T C-M IBI W BS and ${ }^{131}$ scan positive; while the total of ${ }^{131}$ scans positive was 55 (sensitivity: 40,0\%). Eleven patients had ${ }^{131}$ I scan negative and ${ }^{99 m T}$ c-M IBI W BS positive; seven of those proved to be true positive. Although ${ }^{99 m} \mathrm{~T} \mathrm{C-M} \mathrm{IBI} \mathrm{has} \mathrm{low} \mathrm{sensitivity} \mathrm{on} \mathrm{detection} \mathrm{of} \mathrm{tumor} \mathrm{recurrence,} \mathrm{it} \mathrm{may} \mathrm{have} \mathrm{a} \mathrm{role} \mathrm{as}$ a supplementary tool to thyroglobulin measurements and ${ }^{131}$ scans.
\end{abstract}

Key words: Thyroid neoplasms; Carcinoma; Radionuclide imaging; Thyroglobulin; M IBI.

\section{INTRODUÇÃO}

0 carcinoma diferenciado de tireóide (CDT) é um câncer de crescimento lento, caracterizado por evolução favorável devido à disponibilidade de abordagens terapêuticas eficazes: cirurgia, terapia com iodo radioativo ( ${ }^{131} \mathrm{l}$ ) e tratamento hormonal (L-tiroxina) supressivo. 0 prognóstico é excelente nos estágios iniciais da doença, mas pode, também, ser satisfatório mesmo em pacientes que apresentam metástases a distância, particularmente metástases pulmonares (com capacidade de captar ${ }^{131}$ ).

0 tecnécio-99m-metoxiisobutil isonitrila ( ${ }^{99 m} \mathrm{~m}$ c-M IBI), atualmente, utilizado para estudo da função do miocárdio ${ }^{1}$, se mostrou bastante sensível na detecção de tumores; à semelhança do Tálio-201 (201T I), 99mT cM IBI tem sido descrito por se localizar em vários tipos de tumores ${ }^{2,3,4}$. Ele se acumula no tecido tireoideano em vários distúrbios patológicos, como linfoma primário de tireóide ${ }^{5}$, nódulos tireoideanos ${ }^{8}$, carcinoma de células de $\mathrm{H}$ ürthle ${ }^{6}$, câncer medular de tireóde ${ }^{6,7}$, e metástases de $\mathrm{CDT}^{9}$. Em oncologia nuclear, $0{ }^{99 \mathrm{~m} T \mathrm{~T}} \mathrm{C}-\mathrm{MIBI}$ é, amplamente, utilizado na investigação de nódulos mamários não-palpáveis ${ }^{12}$.

$0{ }^{99} \mathrm{mTC}-\mathrm{M} \mid \mathrm{BI}$ se acumula no interior de mitocôndrias e citoplasmas celulares. A carga catiônica e a capacidade lipofílica do ${ }^{99 m} \mathrm{~T}$ c-M IBI, o potencial de membrana plasmática emitocondrial das células tumorais, a quantidade de mitocôndrias nestas células, a perfusão sanguínea aumentada e a permeabilidade capilar são consideradas por desempenhar papel importante no mecanismo de captação tumoral de ${ }^{99 \mathrm{~m} T} \mathrm{c}$-M IBI.
Alguns estudos têm demonstrado que o rastreamento com $99 \mathrm{mT}$ c-M IBI é importante no acompanhamento de pacientes com elevados níveis de tireoglobulina $(\mathrm{Tg})$, mas pesquisa de corpo inteiro ( $\mathrm{PCl}$ ) com ${ }^{131}$ negativo (metástases não-funcionantes mantêm a capacidade de secretar tireoglobulina); ou pacientes com variantes de CDT (ex.: carcinoma de células de H ürthle) que têm pouca avidez por ${ }^{131}$ I. 0 fato do ${ }^{99 \mathrm{mT}} \mathrm{c}$-M IBI detectar tumores não-funcionantes tem grande importância clínica, porque pode direcionar para tratamentos adicionais: radioterapia externa ou excisão cirúrgica.

Valores de tireoglobulina ( $\mathrm{Tg}$ ) sérica e $\mathrm{PCl}$ com radioiodo (131I) têm sido utilizados para seguimento pósoperatório de pacientes com CDT ${ }^{10}$. Em alguns pacientes, o valor da Tg sérica não é confiável devido aos anticorpos anti-tireoglobulina (anti- $\mathrm{Tg}$ ); nestas situações $0{ }^{99 m} \mathrm{~T}$ C-M IBI se mostra importante na tomada de decisão da conduta terapêutica. A cintilografia de corpo inteiro com ${ }^{99 \mathrm{~m} T} \mathrm{c}$-M IBI tem sido usada em aplicação clínica na detecção de metástases de CDT ${ }^{9}$. Seu papel vem sendo considerado como complementar aos valores de $\mathrm{Tg}$ sérica e $\mathrm{PCl}$ com ${ }^{131}$, uma vez que tem se mostrado sensível na detecção de metástases linfonodais, e metástases não-iodocaptantes ${ }^{11}$.

Apesar de muitos estudos terem sido realizados durante o período de hipotireoidismo, alguns autores têm relatado não haver necessidade de suspensão da reposição hormonal ${ }^{2}$. Tal fato torna este exame interessante para uma parcela de pacientes, que não tolera a suspensão da medicação, que é mandatório para a realização de $\mathrm{PCI}$ com 1311; além da suspensão da reposição hormonal favorecer o crescimento tumoral ${ }^{11}$. O utro ponto 
importante, é que a cintilografia com ${ }^{99 \mathrm{~m} T} \mathrm{~T}$ c-M IB I independe da quantidade de tecido tireoideano remanescente; o que não ocorre com o rastreamento de corpo inteiro com ${ }^{131}$. Pacientes que apresentam captação tireoideana acima de $5,0 \%$, não realizam 0 $\mathrm{PCI}$ com ${ }^{131}$ I devido a essa alta captação, fato que limita o rastreamento de metástases em pacientes saídos da cirurgia, e que são candidatos a radioiodoterapia complementar ${ }^{10}$.

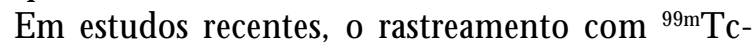
MIBI tem sido comparado com outros métodos de rastreamento de metástases em pacientes com carcinoma diferenciado de tireoide ${ }^{6,13,14}$. 0 rastreamento com ${ }^{99 \mathrm{~m} T \mathrm{c} \text { - }}$ M IBI tem se mostrado altamente sensível na detecção de metástases de CDT, até mesmo mais sensível que $\mathrm{PCl}$ com ${ }^{13112,10 .}$

Alguns autores destacam, ainda, outras vantagens do ${ }^{99 m}$ T c-M IBI em relação ao ${ }^{131}$ I, como por exemplo: a não-necessidade de descontinuar a terapia de reposição hormonal; $0{ }^{99 \mathrm{mT}} \mathrm{T} \mathrm{C}-\mathrm{M}$ IBI produz imagens de melhor qualidade e permite aquisição de tomografia (SPECT); o MIBI é marcado com ${ }^{99 \mathrm{~m} T} \mathrm{c}$, o que permite exame rápido, fácil e com menos exposição a radiação ${ }^{2,10}$.

É importante estabelecer, portanto, qual o papel do M IBI no acompanhamento dos pacientes de CDT através de sua sensibilidade e especificidade na localização precoce de metástases. Este estudo tem como objetivos:

- D eterminar o papel do MIBI em relação aos valores de Tg sérica e ao rastreamento de corpo inteiro com ${ }^{131}$, estudando sua sensibilidade e especificidade em localizar metástases de CDT.

- Estabelecer o melhor tempo para aquisição de imagem pós-injeção do radiofármaco.

\section{PACIENTES, MATERIAL E MÉTODOS}

\section{PACIENTES}

O itenta e nove pacientes com carcinoma diferenciado de tireóide foram avaliados (71 mulheres, 18 homens). A idade foi comprendida entre 13 e 83 anos; média de idade \pm desvio padrão $=46,7 \pm 16,7$. Todos tinham sido previamente submetidos a tireoidectomia total, e receberam dose baixa (diagnóstica) ou alta (terapêutica) de radioiodo 131. Setenta e oito tiveram diagnóstico de carcinoma papilífero (PAP) e 11 de carcinoma folicular (FOL).

Cintilografia com ${ }^{99 \mathrm{mT}} \mathrm{c}$-M IBI foi realizada em todos os pacientes, e os níveis séricos de $\mathrm{Tg}$ foram determinados durante tratamento supressivo (com Ltiroxina) e em hipotireoidismo (4 semanas após suspensão da L-tiroxina). 0 rastremento de corpo inteiro com ${ }^{131}$ f foi feito uma semana após dose terapêutica ou
72 horas após dose diagnóstica.

0 diagnóstico de metástases de CDT foi feito com base em outros métodos diagnósticos, incluindo radiografia simples, tomografia computadorizada (TC), ressonância magnética (RM ), cintilografia óssea e s-T g.

\section{MATERIAL E MÉTOdO}

0 fármaco utilizado foi o hexaquis-metoxiisobutil isonitrila (M IBI) - Cardiolite ${ }^{\circledR}$, produzido pela D uPont Farmacêutica - marcado com o radioisótopo ${ }^{99 \mathrm{~m} T e c n e ́ c i o ~}$ ( ${ }^{99 \mathrm{mT}} \mathrm{T}$ c) obtido através de um gerador de M olibdênio $\left({ }^{99} \mathrm{M} \mathrm{O}\right.$ o) - fabricado pelo Instituto de Pesquisas Energéticas N ucleares (IPEN/SP). Injeção endovenosa em bolus de $740-925 \mathrm{M} \mathrm{Bq}\left(20\right.$ - $25 \mathrm{mC}$ ) do radiofármaco ${ }^{99 \mathrm{mT} \mathrm{C} \text { - }}$ MIBI foi aplicada em uma veia periférica.

Todos os estudos foram realizados na Gama-camâra tomográfica E.CAM com colimador de baixa energia e alta-resolução, e processados em computador ICON P (SIEM EN S M edical Corp.), do Setor de M edicina N uclear do H ospital do Câncer (IN CA). U ma janela de $15 \%$ no canal correspondente a $140 \mathrm{keV}$ foi selecionada no computador de aquisição (ICO N A) da gama-câmara. Imagens de corpo inteiro com matriz de 1024 x 512 foram obtidas de todos os pacientes 20 minutos e 6 horas após a administração do radiofármaco, a uma velocidade de aquisição de $18 \mathrm{~cm} / \mathrm{min}$; em casos selecionados (positivos ou duvidosos) foram obtidas imagens estáticas com matriz de 256 × 256, com tempo de aquisição de 5 - 10 minutos.

Vinte minutos após a administração do radiofármaco é iniciado o estudo cintilográfico (precoce) do paciente; este é colocado em posição supina; o mesmo procedimento é adotado para o estudo cintilográfico de 6 horas (tardia). Para aquisição das imagens estáticas, de uma área de interesse, o paciente é mantido na mesma posição; exceto nos casos de aquisição de imagens oblíquas ou laterais de tórax, em que foi solicitado ao paciente que colocasse o braço em extensão acima da cabeça (de forma confortável).

\section{Interpretação}

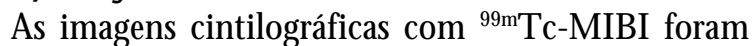
avaliadas por um médico experiente do Setor de M edicina N uclear (IN CA), e classificadas nas seguintes categorias: - Positiva: as que apresentavam hiperfixação anormal do radiofármaco, em um local do corpo.

- N egativa: as que apresentavam distribuição fisiológica do radiofármaco no organismo.

\section{Análise dos resultados}

As imagens positivas foram dividas de acordo com a possível localização da lesão: pescoço (restos 
glandulares ou linfonodo); pulmão; osso ou linfonodo (extra-cervical). Esta classificação foi necessária para a comparação do estudo cintilográfico com ${ }^{99 \mathrm{mT}} \mathrm{c}-\mathrm{M}$ IBI e 0 rastreamento de corpo inteiro com ${ }^{131}$; ; e, também, com os valores de Tg sérica, tanto em tratamento supressivo quanto em hipotireoidismo.

O s níveis séricos de $\mathrm{Tg}$ foram determinados durante tratamento supressivo com L-tiroxina e 4 semanas após suspensão do hormônio tireoideano. O s valores de Tg foram considerados positivos quando atingiram níveis superiores a $2 \mathrm{ng} / \mathrm{ml}$, em supressão hormonal ou em hipotireoidismo.

\section{Confirmação de doença residual}

O s casos discordantes seguiram maior investigação com outros métodos diagnósticos (radiografia simples, TC, etc.) para confirmação ou exclusão (verdadeiro positivo ou falso positivo, respectivamente). $\mathrm{N}$ os pacientes com rastreamento com ${ }^{131}$ | negativo e $\mathrm{Tg}$ elevada (em hipotireoidismo) radiografia simples, TC, RM, ultrassonografia ou biópsia foram realizados, de acordo com a necessidade.

0 rastreamento com ${ }^{131}$ | pós-dose terapêutica foi considerado o padrão-ouro para confirmação de câncer recidivante ou metastático.

\section{Análise estatística}

O s resultados foram classificados como verdadeiros positivos (VP) ou falso positivos (FP) quando a cintilografia com ${ }^{99 \mathrm{mT}} \mathrm{T}$ c-M I BI era confirmada ou não por outro método

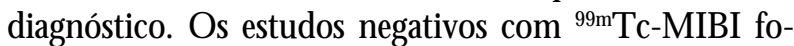
ram definidos como verdadeiros negativos ou falso negativos de acordo com a confirmação ou exclusão de doença residual em atividade, respectivamente.

A sensibilidade, especificidade, valor preditivo positivo (VPP) e valor preditivo negativo (VPN) foram calculados. N este estudo são usadas, somente, variáveis em escala nominal: positiva ou negativa; presença de câncer ou ausência de câncer. Por isso foi usado o teste do Chi-quadrado, para independência na avaliação dos resultados; e determinar se a freqüência de captação de 99mT c-M IBI é significativa em comparação com a captação de ${ }^{131}$. 0 nível de significância foi fixado em 5 centésimos $(p \varangle 0,05)$, para o teste do Chi-quadrado.

\section{RESULTADOS}

A faixa etária dos pacientes variou entre 13 e 83 anos (Figura 1), com predomínio da 5ạ década. A média de idade deste estudo foi de 46,7 anos.

D entre os 89 pacientes estudados, 71 (79,7\%) eram mulheres e $18(20,3 \%)$ eram homens. 0 tipo histopatológico mais freqüente foi o carcinoma papilífero (87,6\%); enão foi observado tipo histológico predominante em relação ao sexo do paciente (figura 2).

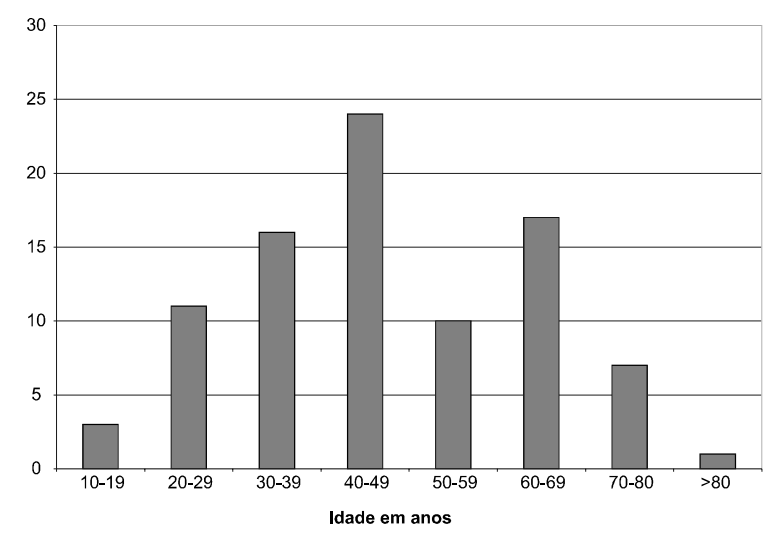

Figura 1: Distribuição dos 89 casos estudados em relação a faixa etária

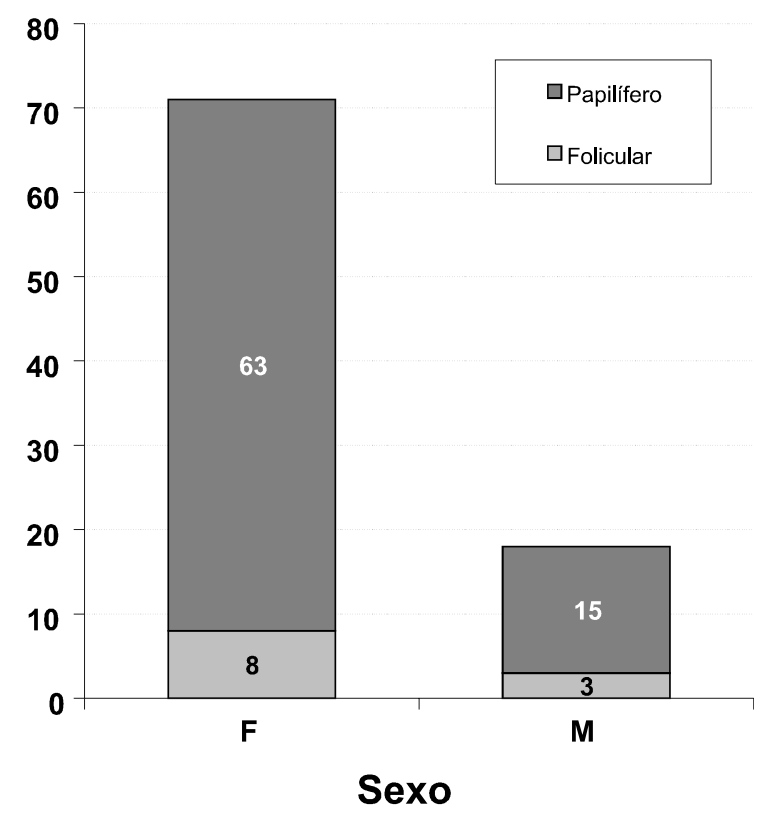

Figura 2: Distribuição do tipo histopatológico do câncer de tireóide em relação ao sexo dos pacientes

O s resultados dos achados cintilográficos com ${ }^{99 \mathrm{mT}} \mathrm{c}$ MIBI foram classificados como pescoço, linfonodo, pulmão ou 0sso, de acordo com sua localização para melhor análise dos resultados.

Vinte e três $(25,8 \%)$ dos 89 pacientes estudados apresentaram rastreamento com ${ }^{131}$ e cintilografia com 99mT c-M IBI negativas. N este grupo 6 pacientes apresentaram Tg sérica el evada, em tratamento supressivo e em hipotireoidismo, e 2 pacientes apresentaram Tg positiva somente após suspensão da L-tiroxina. 
D os 23 pacientes que apresentaram rastreamento com ${ }^{131}$ | negativo, 7 tinham câncer recidivante ou metastático, 4 destes pacientes foram descobertos pela cintilografia com ${ }^{99 m}$ T c-MIBI, 2 com metástase linfonodal e 2 com metástases pulmonares (Figura 3). $\mathrm{N}$ os outros 3 pacientes, o câncer residual foi detectado pela s-Tg elevada e outros métodos diagnósticos, e confirmado com dose terapêutica de ${ }^{131}$ adicional.

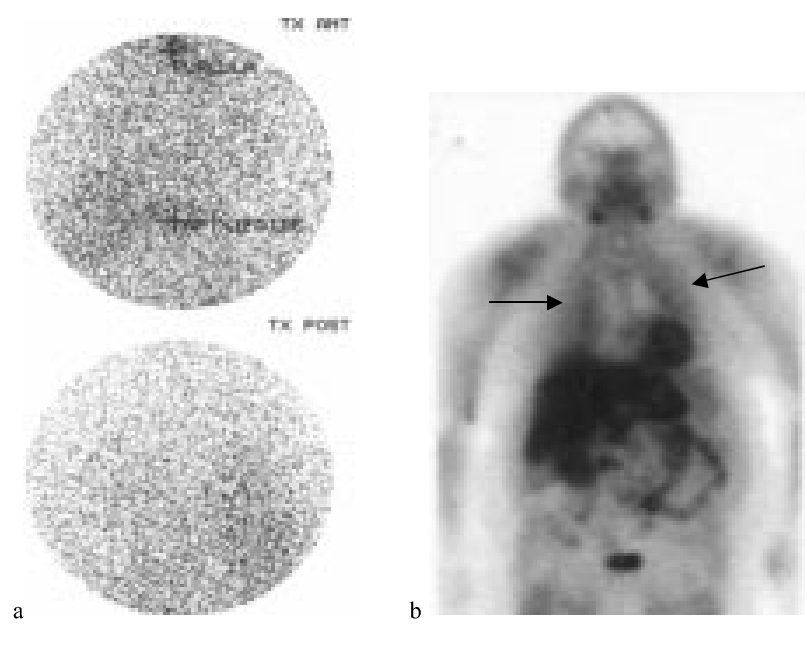

Figura 3: Caso 84. a- Rastreamento com ${ }^{131}$ negativo, e bCintilografia com ${ }^{99 \mathrm{~m} T \mathrm{C}-\mathrm{MIBI} \text { positiva. }}$

O s níveis séricos de T $\mathrm{g}$ foram determinados durante o tratamento supressivo com L-tiroxina e após 4 semanas de suspensão do hormônio tireoideano antes da dose diagnóstica ou terapêutica de ${ }^{131}$, que evidenciou $55 \%$ (12/22) dos pacientes com rastreamento com ${ }^{131}$ I negativo tinham $\mathrm{Tg}$ positiva. Cinco destes pacientes apresentavam $\mathrm{Tg}$ elevada em hipotireoidismo e sem evidências radiológicas de recidiva tumoral. Apesar de 7 pacientes apresentarem $\mathrm{Tg}$ elevada durante tratamento supressivo e em hipotireoidismo, câncer recidivante ou metastático foi detectado em 4 pacientes pela cintilografia com ${ }^{99 \mathrm{mT}} \mathrm{C}$-M IBI; os outros 3 pacientes apresentaram captação anormal, somente, visualizada após tratamento adicional com radioiodo.

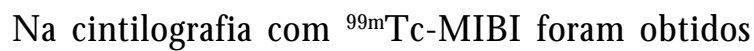
resultados positivos em 33 pacientes (37\%). Vinte e dois pacientes tiveram resultados concordantes com 0 rastreamento com ${ }^{131}$; d dos 11 pacientes que tiveram resultados discordantes 8 foram confirmados positivos por outros métodos diagnósticos (Raios- $X$ simples, IRM, TC ou USG); os demais se mostraram serem falsos positivos.

Vinte e dois pacientes apresentaram rastreamento com ${ }^{131}$ positivo, e cintilografia com ${ }^{99 m}$ T C-M IBI negativa. D estes, 5 pacientes apresentavam captação pulmonar e 2 em estruturas ósseas e 1 paciente apresentava captação pulmonar e em região cervical, e 14 pacientes foram classificados como positivos em região cervical (pescoço). N este grupo, 16 pacientes apresentavam Tg elevada, 1 paciente apresentava Tg e anticorpo anti-T g positivos e captação pulmonar do radioiodo.

A sensibilidade e especificidade do ${ }^{99 m}$ T C-M IB I encontradas foram de $40 \%$ e $67,6 \%$, respectivamente (Tabela 1), e os valores preditivos positivo e negativo, respectivamente, foram $66,6 \%$ e $41 \%$. Em se considerando os verdadeiros positivos detectados pela cintilografia com ${ }^{99 m}$ T C-M IBI e não observados no rastreamento com ${ }^{131}$, a sensibilidade passa a ser de $66,7 \%$ e a especificidade de $86,7 \%$ (Tabela 2).

Tabela 1: Comparação entre cintilografia com ${ }^{99 m}$ Tc-MIBI e rastreamento com |-131

\begin{tabular}{c|c|c|c}
\hline & \multicolumn{2}{|c|}{ MIBI } \\
\hline I-131 & Negativo & Positivo & Total \\
\hline Negativo & 23 & 11 & 34 \\
\hline Positivo & 33 & 22 & 55 \\
\hline Total & 56 & 33 & 89 \\
\hline$(p=0,468)$ & \multicolumn{3}{l}{}
\end{tabular}

Tabela 2: Comparação da cintilografia com ${ }^{99 m} \mathrm{mc}-\mathrm{MIBI}$ e rastreamento com ${ }^{131}$ | e tireoglobulina $(\mathrm{s}-\mathrm{Tg})$

\begin{tabular}{|c|c|c|c|}
\hline s-Tg supr & \multicolumn{2}{|c|}{$99 m T c-M|B|$} & $\begin{array}{l}\text { Significância } \\
\text { estatística }\end{array}$ \\
\hline A & Positivo & Negativo & \\
\hline $0-2$ & 13 & 34 & $\mathrm{P}=0,051^{*}$ \\
\hline \multirow[t]{2}{*}{$>2$} & 20 & 22 & \\
\hline & \multicolumn{2}{|c|}{${ }^{131} \mid$} & \\
\hline B & Positivo & Negativo & \\
\hline $0-2$ & 24 & 23 & $P<0,05$ \\
\hline$>2$ & 31 & 11 & \\
\hline
\end{tabular}

* Sem associação

$\mathrm{Na}$ tabela 2 são comparados os achados cintilográficos do estudo com ${ }^{99} \mathrm{mT} \mathrm{c}-\mathrm{M}$ IBI e 0 rastreamento com radioiodo em relação aos valores de tireoglobulina em supressão.

N este estudo, adotamos a aquisição de imagens 20 minutos (precoces), 6 horas e 24 horas (tardias) após a injeção de ${ }^{99 m T ~ c-M ~ I B I, ~ p a r a ~ a v a l i a c ̧ a ̃ o ~ d o ~ m e l h o r ~ t e m p o ~}$ de aquisição das imagens. Cintilografias com ${ }^{99 m} \mathrm{~T}$ c$\mathrm{M}$ IBI precoce e tardia foram comparadas em todos os pacientes. Durante o trabalho não foi observada 
nenhuma nova lesão nas imagens tardias ( $6 \mathrm{~h}$ e $24 \mathrm{~h}$ ); somente $25 \%$ das alterações cintilográficas observadas nas imagens de 20 minutos (precoces) se mantiveram nas imagens tardias; que foram confirmadas por método complementar de imagem. Foi observado, ainda, que, nos casos concordantes, a cintilografia com ${ }^{99 m T}$ c-M IB I apresenta melhor definição da área comprometida que o rastreamento com ${ }^{131}$ I (figura 4). Esta observação está de acordo com os trabalhos pesquisados, que adotam somente as imagens precoces ou imagens de até duas horas (tardias).

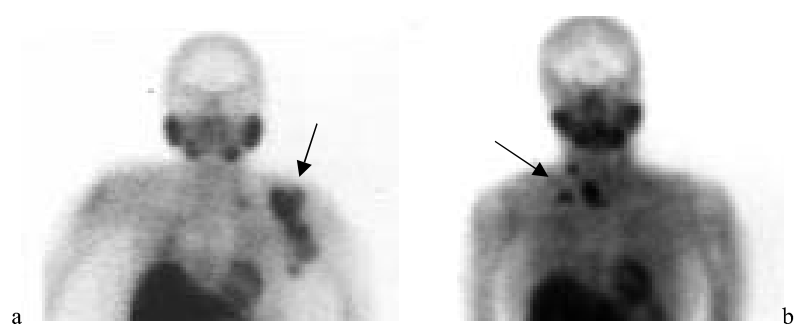

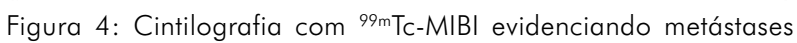
ósseas (a) e linfonodais (b).

D os que apresentavam alteração cintilográfica na imagem precoce $(n=33)$, somente $18 \%$ (6 pacientes) mantiveram as alterações $6 \mathrm{~h}$ após a injeção do radiofármaco. Trinta pacientes foram confirmados por outros métodos diagnósticos; destes, seis pacientes mantiveram captação anormal nas imagens tardias, e apresentavam metástases ósseas ou linfonondais. Três pacientes apresentavam alterações osteoarticulares, confirmadas por método complementar, e foram considerados falsos positivos. Foram considerados positivos os exames que apresentaram captação anormal em, pelo menos um rastreamento. $\mathrm{N}$ ão foi observada nenhuma nova alteração nas aquisições tardias.

\section{DISCUSSÃO}

Apesar do câncer diferenciado de tireóide (CDT) ser uma doença de evolução lenta e reservar para a maioria dos pacientes uma expectativa de vida normal, a recidiva tumoral ou a doença metastática apresenta grande morbidade. Acompanhamento de longo-prazo e tratamento suplementar são necessários. Em vista disto, é mais importante o monitoramento dos pacientes que apresentam fatores prognósticos ruins ao diagnóstico, especialmente para recidiva local ou metástases à distância ${ }^{15}$.

A sensibilidade do rastreamento com ${ }^{131}$ n no CDT tem sido observada em torno de $84 \%$, com uma alta especificidade $90 \%$ - 100\%; o rastreamento com ${ }^{131}$ I é o principal método diagnóstico, e indispensável na avaliação para tratamento complementar ${ }^{15}$. 0 utra importante informação na avaliação do CDT é a Tg, que é produzida pelas células foliculares tireoideanas e não é detectável no plasma de indivíduos atireoi deanos ${ }^{16}$. Porém, o rastreamento com ${ }^{131}$ e a s-Tg tem desvantagens como a pouca sensibilidade em pacientes com restos tireoideanos, al ém da necessidade da suspensão da terapia supressiva com hormônio tireoideano $0^{9,16,17}$.

O ${ }^{99 m}$ T c-M IBI, um complexo molecular catiônico, foi introduzido como um fármaco para avaliação da perfusão miocárdica, mas sua semelhança com o Tálio$201{ }^{201} \mathrm{TI}$ ) permitiu que fosse avaliado como fármaco

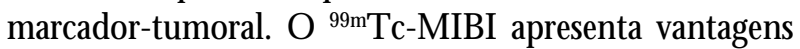
sobre $0{ }^{201} \mathrm{TI}$, principalmente, porque é facilmente disponível e gera imagens de melhor qualidade $e^{4,5}$.

$\mathrm{N}$ o presente estudo, a cintilografia de corpo inteiro com ${ }^{99 m}$ T c-M IBI apresentou baixa sensibilidade (42\%) e alta especificidade (87\%) para detecção de câncer de tireóide recidivante ou metastático. A comparação direta

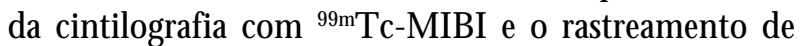
corpo inteiro com ${ }^{131}$ | revelou baixa sensibilidade $(36,8 \%)$ ealta especificidade (72,7\%). Em outros estudos que avaliaram $0{ }^{99 m} \mathrm{~T} \mathrm{C}$-M IBI em CDT, $\mathrm{Ng}$ et al. ${ }^{18} \mathrm{e}$ $D$ adpavar et al. ${ }^{19}$ observaram que $0{ }^{99 \mathrm{~m}} \mathrm{~T} \mathrm{C}$-M IBI é menos sensível que $0{ }^{131}$. 0 último apresentou como resultado sensibilidade $(36 \%)$ semel hante mas especificidade $(89 \%)$ mais alta que o encontrado no nosso estudo.

Roelants et al. ${ }^{9}$ sugerem que um rastreamento de corpo inteiro com ${ }^{131} \mid$ associado a $\mathrm{Tg}$ elevada pode ser explicado por problemas técnicos (como limitação da resolução espacial pelo sistema de detecção ou contaminação com iodo não-radioativo) ou por indiferenciação das células neoplásicas. Fatourechi e $\mathrm{H}$ ay ${ }^{20}$ em estudo de revisão sobre rastreamento com ${ }^{131}$ I negativo eT g elevada observaram que o rastreamento com ${ }^{131}$ pós-dose ablativa ou terapêutica pode mostrar tecido iodocaptante ectópico em $24 \%$ dos pacientes com níveis de $\mathrm{Tg}$ entre 6 - 15ng/ml e, quando a Tg é maior que $15 \mathrm{ng} / \mathrm{ml}$, captação ectópica pode ser vista em $46 \%$ dos pacientes.

$\mathrm{N}$ g et al. ${ }^{18}$ relatam que $96 \%$ dos pacientes com $\mathrm{Tg}$ inicial (pós-tireoidectomia) maior que $30 \mathrm{ng} / \mathrm{ml}$ (em hipotireoidismo) têm evidência cintilográfica de remanescente tireoideano, comprometimento linfonodal ou metástases à distância, e a cintilografia com ${ }^{99 \mathrm{mT}} \mathrm{c}$ M IBI foi positiva em $83,5 \%$ desses pacientes. $N$ o presente estudo, 89\% dos pacientes com restos glandulares ou metástases à distância apresentaram $\mathrm{Tg}$ elevada (acima de $15 \mathrm{ng} / \mathrm{ml}$ ) em hipotireoidismo, e a cintilografia com 99mT c-M IBI foi positiva somente em $36 \%$ destes pacientes. Eles observaram que $44 \%$ dos pacientes com 
s-T g menor que 30 $\mathrm{ng} / \mathrm{ml}$ tinham evidência cintilógrafica de câncer residual ou metastático, e que a cintilografia com ${ }^{99 m}$ T c-M IBI detectou $71,7 \%$ destes pacientes; contrariando os dados obtidos em nosso estudo.

O bservamos que dos 26 pacientes com rastreamento com ${ }^{131}$ positivo, vinte e cinco apresentaram s- $\mathrm{Tg}$ positiva; a cintilografia com $99 \mathrm{mT}$ c-M IBI foi positiva somente em 10 desses pacientes (38,4\%); a cintilografia com ${ }^{99 \mathrm{mT}}$ c-M IBI falhou em mostrar captação cervical, metástases pulmonar e óssea. $\mathrm{N}$ o entanto, a cintilografia com ${ }^{99 \mathrm{mT}} \mathrm{C}$-M IBI foi positiva em um paciente com metástase pulmonar e, ambos, rastreamento com ${ }^{131}$ e s-T g negativos, mas anticorpo anti-tireoglobulina positivo.

Em nosso estudo encontramos evidência cintilográfica de câncer recidivante ou metastático em 30 pacientes $(n=48$ ) e outros 3 pacientes com T g positiva foram confirmados após nova dose terapêutica de radioiodo. Entretanto, 5 pacientes com cintilografia com ${ }^{99 m}$ T C-M IBI e rastreamento com ${ }^{131}$ | negativos apresentaram T g elevada durante suspensão da L-tiroxina (valores entre 10 - 30 $\mathrm{ng} / \mathrm{ml}$ ); estes pacientes foram submetidos a investigação complementar com outros métodos diagnósticos e não foi observada evidência de doença em atividade; isto poderia ser atribuído à produção de tireoglobulina por lesões muito pequenas, não detectáveis por métodos convencionais de imagem. Roelants et al. ${ }^{9}$ em estudo sobre 0 valor preditivo da $\mathrm{Tg}$ no acompanhamento de pacientes com CDT sugerem que a Tg apresente uma curva decrescente após 0 tratamento com radioiodo por um período variável. Fatourchi e $\mathrm{H} \mathrm{ay}{ }^{20}$ relatam que pacientes com CDT e metástases pulmonares não vistas na radiografia simples de tórax têm uma possibilidade de 5\% de estar associada a níveis indetectáveis durante terapia supressiva com Ltiroxina, enquanto linfonodos cervicais têm $20 \%$ de chance de ocorrer o mesmo. No entanto, observamos não haver associação estatística entre a s-T g (em supressão) e a cintilografia com ${ }^{99 \mathrm{~m} T} \mathrm{~T}-\mathrm{M}$ IBI $(p=0,051)$.

Alguns estudos comparando cintilografia com ${ }^{99 \mathrm{mT}} \mathrm{C}$ $\mathrm{M} I \mathrm{BI}$ e rastreamento com ${ }^{131}$ I têm apresentado resultados divergentes. Alam et al. ${ }^{10}$ continuando 0 estudo de $M$ iyamoto et al. ${ }^{2}$ relatam que a cintilografia com ${ }^{99 \mathrm{mT}} \mathrm{c}$ M IBI tem alta sensibilidade $(87,8 \%)$ e especificidade $(95,6 \%)$, e sugere a cintilografia com ${ }^{99 m}$ T c-M IBI como método cintilográfico de escolha na avaliação de pacientes com CDT. Roelants e Beckers ${ }^{21}$, em carta ao editor referindo-se ao estudo de M iyamoto et al. ${ }^{2}$, relatam que, em sua experiência, a cintilografia com ${ }^{99 m}$ T c-M IBI foi positiva em, apenas, $64 \%$ de 31 pacientes com rastreamento com ${ }^{131}$. D adpavar et al. ${ }^{18}$ encontraram uma baixa sensibilidade (36\%) mas alta especificidade (89\%) para a cintilografia com ${ }^{99 m} \mathrm{~T}$ cM IBI quando comparada com o rastreamento com ${ }^{131}$.

Em trabalho recente, I wata et al. ${ }^{22}$ comparam a tomografia por emissão de pósitrons (PET) com ${ }^{18} \mathrm{~F}$ FD G com rastreamento com ${ }^{131}$ e a tomografia por emissão e fóton único (SPECT) de corpo inteiro com 99mT c-M IBI e demonstraram que o SPECT aumenta a sensibilidade do exame com ${ }^{99 m} \mathrm{~T}$ c-M IBI; e há correlação da cintilografia com ${ }^{99 \mathrm{~m} T \mathrm{~T}} \mathrm{C}-\mathrm{M}$ IBI e o PET com ${ }^{18} \mathrm{~F}-\mathrm{FD} \mathrm{G}$. I wata et al. ${ }^{22}$ não encontraram associação entre o SPECT com ${ }^{99 \mathrm{~m} T} \mathrm{C}-\mathrm{M}$ IBI e o rastreamento com ${ }^{131}$, entretanto sugeriram ser importante a realização dos exames diagnósticos funcionais serem realizados com o paciente em hipotireoidismo.

$\mathrm{N}$ ossos resultados confirmam os achados de outros, que 0 rastreamento com ${ }^{131}$ é mais sensível que a cintilografia com ${ }^{99 m} \mathrm{~T}$ c-M IBI para identificação de focos de captação na região cervical e em pulmões, apesar da cintilografia com ${ }^{99 \mathrm{mT}} \mathrm{T}$ c-M IBI detectar mais metástases linfonodais e delimitar melhor lesões ósseas que 0 rastreamento com ${ }^{131}$.

O s trabal hos realizados com ${ }^{99 \mathrm{~m} T} \mathrm{~T}$ c-M IBI na detecção de recidiva ou metástases de CDT apresentam resultados divergentes devido ao fato de pesquisadores adotarem critérios variados para a positividade da tireoglobulina. Em nosso trabalho optamos pela referência com os menores valores, pois níveis indetectáveis de tireoglobulina são um sinal de ausência de tecido tireoideano.

Podemos observar, na casuística estudada, que 0 99mT c-M IBI pode desempenhar papel importante em casos de tireoglobulina elevada, e em casos de anticorpo anti-tireoglobulina positivo e rastreamento com ${ }^{131}$ negativo. Embora não haja uma correlação entre níveis elevados de tireoglobulina (em supressão) ea cintilografia com ${ }^{99 m T} \mathrm{c}-\mathrm{M}$ IBI $(p=0,051)$, parece haver uma tendência neste sentido, que poderia se tornar evidente caso fossem excluídos os pacientes com doença em estágio inicial. Porém, é necessário maior número de casos para uma conclusão definitiva nesse sentido.

Vale ressaltar que não foram encontrados, na literatura, trabalhos que avaliassem todos os pacientes em controle de CDT; os trabalhos disponíveis na literatura investigam os pacientes suspeitos ou sabidamente com doença metastática.

\section{CONCLUSÕES}

Em vista aos resultados obtidos nas cintilografias com 99mT c-M IBI de 89 pacientes com câncer diferenciado de tireóide (CDT), correlacionados com rastreamento com ${ }^{131}$ e os valores séricos de tireoglobulina, pode-se 
concluir que:

- $0{ }^{99 m}$ T c-M IBI é pouco sensível $(40 \%)$ e pouco específico $(67,7 \%)$ em comparação ao rastreamento com 131., na detecção de tecido residual ou metastático. 0 $99 \mathrm{mT}$ c-M IBI tem melhor sensibilidade $(66,7 \%)$ e alta especificidade $(86,7 \%)$ quando correlacionado a tireoglobulina sérica (em supressão), porem sem associação estatisticamente significativa ( $P=0,051)$.

- 0 melhor tempo para realização da aquisição de imagem cintilográfica com ${ }^{99 m}$ T c-M IBI é 20 minutos após a injeção; imagens tardias não acrescentam nova informação.

\section{REFÊRENCIAS}

1. Rigo P, Braat SH . Radiopharmaceuticals for the study of the heart. In: M urray IPC, EII PJ. N uclear medicine in clinical diagnosisand treatment. 2nd ed. LW W; 1998. p. 1309-18.

2. M iyamoto S, Kasagi K, M isaki T, Alam M S, Konishi J. Evaluation of Technetium-99m-M IBI scintigraphy in metastatic differentiated thyroid carcinoma. J N ucl M ed. 1997;38:352-6.

3. Spencer RP. Tumor-seeking radiopharmaceuticals: nature and mechanisms. In: M urray IPC, Ell PJ. N uclear medicinein clinical diagnosisand treatment. 2aed. LWW; 1998. p. 759-74.

4. H assan IM, Sahweil A, Constantinides C, M ahmoud A, $\mathrm{N}$ air M , O mar YT, et al. U ptake and kinetics of $99 \mathrm{~m}-\mathrm{T} \mathrm{c}$ hexakis-2-methoxiisobutilisonitrilein benign and malignant lesionsin thelungs. Clin N ucl M ed. 1989;14:333-40.

5. Aktolun C, Bayhan H, Kir M . Clinical experience with $99 \mathrm{mT}$ c-M IBI imaging in patientswith malignant tumours. Preliminary resultsand comparison with 201T I. C lin N ucl M ed. 1992;17:171-6.

6. Casara D, Rubello D, Saladini G, M azzarotto R, Sotti G, TomasellaG, et al. Clinical approach in patients with meta static differentiated thyroid carcinoma and negative 131-I wholebody scintigraphy: importance of $99 \mathrm{mT}$ c-M IBI scan combined with high resolution neck ultrassonography. Tumori. 1999;85:122-7.

7. Costa M GF, CostaFilho CFF, M oura LA Jr. Literature re view of the use of scintimammography with technetium99m methoxyisobutylisonitrile (99mT c-M IBI) for thedifferential diagnosis of breast carcinoma. Rev Bras Cancerol. 2001;47:33-42.

8. Ramanna $L$, Waxman $A D$, Brachman $M B$, Sensel $N$, Tanasescu D E, Berman DS, et al. Correlation of thyroglobulin measurements and radio iodinescansin the followup of patients with differentiated thyroid cancer. $C$ ancer. 1985;55:1525-9.

9. RoelantsV, D eN ayer P, Bouckaert A, Beckers C. The pre dictive value of serum thyroglobulin in the follow-up of differentiated thyroid cancer. Eur J Nucl Med.
1997;24:722-7.

10. Alam M S, Kasagi K, M isaki T, M iyamoto S, I wata M , Iida $Y$, et al. Diagnostic value of technetium-99m methoxyisobutyl isonitrile (99mT c-M IBI) scintigraphy in detecting thyroid cancer metastases: a critical evaluation. Thyroid. 1998;8:1091-100.

11. RiccabonaG . D ifferentiated thyroid carcinoma. In: M urray IPC, EII PJ. N uclear medicinein clinical diagnosisand treatment. 2a ed. LW W; 1998. p. 941-58.

12. Goldsmith SJ. Thyroid carcinoma. In: K halkhali I, M aublent JC, Goldsmith SJ. N uclear oncology. LW W; 2001. p. 197-220.

13. Sarikaya A, H useyinova G, I rfanoglu M E, Erkmen N, ÇermikT F, BerkardaS. Therelationship between $99 \mathrm{mT} \mathrm{c}$ sestamibi uptakeand ultrastructural cell types of thyroid tumours. N ucl M ed Com. 2001;22:39-44.

14. Rubello D, M azzarotto R, C asara D. The role of technetium-99m methoxyisobutylisonitrile scintigraphy in the planing of therapy and follow-up of patients with differentiated thyroid carcinoma after surgery. Eur] $\mathrm{N}$ ucl $\mathrm{M}$ ed. 2000;27:431-40.

15. $H$ augen $B R$, Lin EC. I sotope imaging for metastatic thyroid cancer. Endocrinol $\mathrm{M}$ etab $\mathrm{Clin} \mathrm{N}$ orth $\mathrm{Am}$. 2001;30:469-92.

16. Spencer CA, LoPresti JS, Fatemi S, N icoloff JT. D etection of residual end recurrent differentiated thyroid carcinoma by serum thyroglobulin measurement. Thyroid. 1999;9:435-41.

17. Spencer CA, WangCC. Thyroglobulin measurement: techniques, clinical benefits and pitfalls. Endocrinol $M$ etab N orth Am. 1995;24:841-64.

18. N g D CE, Sundram FX, Sin AE. 99mT c-Sestamibi and 131 whole body scintigraphy and initial serum thyroglobulin in the management of differentiated thyroid carcinoma. J N ucl M ed. 2000;41:631-5.

19. D adpavar S, ChevresA, Tulchinsky M , K rishna-Badrinath $L$, K han AS, Sizovsky W J. Clinical utility of technetium99m methoxisobutylisonitrile imaging in differentiated thyroid carcinoma: comparison with thallium-201 and iodine-131 scintigraphy and serum thyroglobulin quantitation. EurJ N ucl M ed. 1995;22:1330-8.

20. Fatourechi $V, H$ ay ID. Treating the patient with differentiated thyroid cancer with thyrogloblin-positiveiodine-131 diagnostic scan-negativemetastases: including comments on the roleof serum thyroglobulin monitoring in tumour surveillance. Semin N ucl M ed. 2000;2:107-14.

21. RoelantsV, BeckersC. 99mT c-M IBI in differentiated thyroid carcinoma. J N ucl M ed. 1999;40:1769.

22. I wataM , Kasagi K, M isaki T, M atsumoto K, lidaY, Ishimori $\mathrm{T}$, et al. Comparison of whole-body 18F-FDG PET, 99mT c-M IBI SPECT and post-therapeutic 131I-N a scintigraphy in the detection of metastatic thyroid cancer. Eur J N ucl M ed M ol I maging. 2004;31:491-8. 